C-A/AP/\#141

March 2004

\title{
CONCEPTS for CAPACITIVELY RF- SHIELDED BELLOWS in cryogenic structures
}

\author{
Y. Zhao, H. Hahn
}

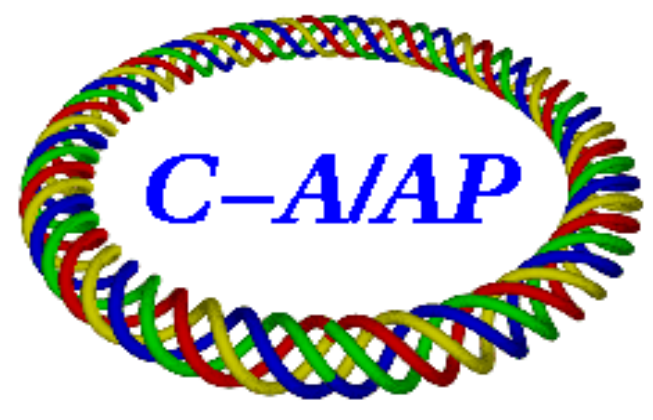

Collider-Accelerator Department Brookhaven National Laboratory

Upton, NY 11973 


\title{
CONCEPTS for CAPACITIVELY RF-SHIELDED BELLOWS in cryogenic structures
}

\author{
Yongxiang Zhao, Harald Hahn \\ Brookhaven National Laboratory, Upton, NY 11973
}

\begin{abstract}
Bellows are frequently required in accelerators and colliders. Usually RFshields with spring fingers are employed to screen the bellows. The lack of accessibility in cryogenic systems can be a problem and asks for alternate solutions to eliminate possible overheating, sparking, etc that occurred in intensive beams. This note addresses an alternate kind of RF shield, which uses capacitive contact instead of mechanical contact. The analysis, as well as numerical example of a superconducting cavity structure, shows that the capacitive RF shield satisfies the impedance requirements of both beam and HOMs. The capability of thermal isolation is also analyzed.
\end{abstract}

\section{INTRODUCTION}

In accelerators and storage rings, bellows are frequently required between connecting beam pipes to provide the necessary working space in response to thermal expansion and contraction. They also provide the necessary tolerances for misalignment. Besides, in the case of a cryostat containing a superconducting cavity, like that for the "Energy Recovering Linac (ERL)" experiment at BNL [1,2], a bellows is also considered as a thermal isolation to reduce the heat load of the cryogenic system.

However, the bellows introduces an added impedance to the beam and provokes high order modes (HOM), which are harmful to the beam stability [3]. To this end, RFshielded bellows are usually employed to reduce the impedance and suppress the HOMs.

The most common RF shield is of the finger type structure, which shields the RF field and is movable longitudinally to keep pace with the stretch of the bellow. The structure consists of many narrow and thin fingers distributed azimuthally on the inner surface of the beam pipe [4,5].

This kind of structure was successfully used in many accelerators. However, as the state-of-the-art advanced, the accelerators and colliders required higher and higher luminosity, obtained from high beam current and short bunch length. As a result, the RFshields as well as the bellows have encountered problems such as overheating, leakage or even damage [6,7].

There are two problems in the finger type structure. The finger has slots which can not completely shield the field, especially for TE-like modes, in which the azimuthal current component is interrupted by the slots. It turns out that the field couples to the bellows chamber causing troublesome HOMs. Secondly, the finger contact can not be perfect. Sliding fingers in principle have only limited points being really contacting. All current flows through those points with very high density, which results in local overheating, or sparking in a more serious case. Besides, the fingers may also produce dust inside the beam pipe due to friction. Although some improvements have been made 
in new structures, which alleviated the problems, none has completely avoided the mechanical contacts [8]. Replacement of bellows is rendered more difficult in a cryogenic environment and effectively precludes spring finger designs.

This note addresses a bellows structure with a capacitive RF shield, i.e. without any mechanical contact. It seems promising to overcome the above problems and could be used at superconducting cavities.

\section{BEAM IMPEDANCE OF A CAPACITANCE}

When a bunched beam travels along the beam line, it induces its image charge which travels along the inner surface of the beam pipe. If the pipe is uniform, the image charge will encounter little resistance. The wake field is also uniform with a simple pattern. Any non-uniformity, like a bellows, will perturb the field. This field can be regarded as combination of many high order modes, provided the system is linear which is true for most cases. Those modes will in turn react on the beam, especially if a resonance occurs.

To deal with those modes usually involves complex mathematics. Nevertheless, the basic concept is rather simple. The image charge across a non-uniformity, say a bellows, will establish a voltage followed by a decaying oscillation.

Suppose a capacitor is connected across the bellows, the image charge is then accumulated in the capacitance with a voltage being established. Once the bunch passes the capacitance, the accumulated charge will discharge through the bellows and form a decaying oscillation. For simplicity, the bellows can be represented by an inductance. Fig.1 shows the schematic equivalent circuit.

Assuming the size of the capacitor is negligible in comparison with the bunch length, one can treat a single bunch as a lumped charge, $q$. The voltage established on the capacitance is simply:

$$
V=q / C
$$

As known, the bunch sees only onehalf of its own beam induced field [9], namely, it is decelerated by a voltage of

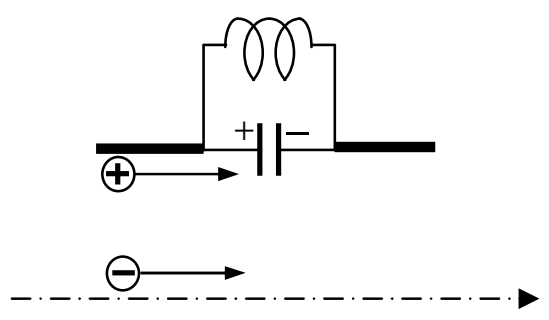

Fig.1. The schematic of the equivalent circuit of the capacitive RF shield

$$
V_{e}=V / 2
$$

This argument can easily be justified by the energy conservation law. Because the energy stored in the capacitance is

$$
W=1 / 2 C V^{2} .
$$

It must be equal to the energy lost by the bunch. The latter is

$$
W_{l}=V_{e} \cdot q=V_{e} \cdot C V
$$

By equating the above two formulas, we get Eq. (2) immediately. Thus the loss factor is: 


$$
k=\frac{W_{l}}{q^{2}}=\frac{1}{2 C}
$$

The capacitance can be made large, so that the loss factor can be quite small.

Suppose that the bunches are delivered with the repetition rate, $f$, but that the interval between two bunches is long enough so that the oscillation vanishes before the arrival of the next bunch, then the total loss per unit time is:

$$
P=f W_{l}=f \frac{q^{2}}{2 C}
$$

One may justifiably argue that Fig. 1 is too simple for generating HOMs. If one seeks for an equivalent circuit with HOMs, the inductance should be replaced by a much more complex circuit. Yet, it will not change the basic phenomenon except if a resonance occurs. To depress HOMs is certainly important and it will be addressed later.

\section{CAPACITIVE RF-SHIELD STRUCTURE}

A capacitor can be formed by two coaxial cylinders with a gap in between. Fig. 2 shows the schematic. This capacitor also functions as a RF-shield. Intuitively, the beam is completely screened from the bellows, because there is no slot between beam pipe and bellows chamber. When the bellows is stretched or compressed, the capacitance will change slightly without any mechanical friction. Thus it avoids the disadvantages of the finger type RF shield.

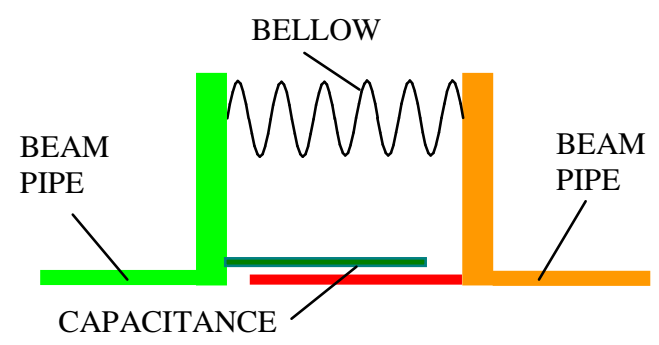

BEAM AXIS

Fig. 2 Capacitor formed by two coaxial cylinders: Case 1

As shown in equation (5), the larger the capacitance, the smaller the loss factor. To this end, one would prefer a larger one. There are many possible methods to do so. For example, one may insert a high dielectric constant material in the gap. One may also increase the area of the electrodes. Fig.3 shows a structure having a number of teeth on the cylinders [8]. 

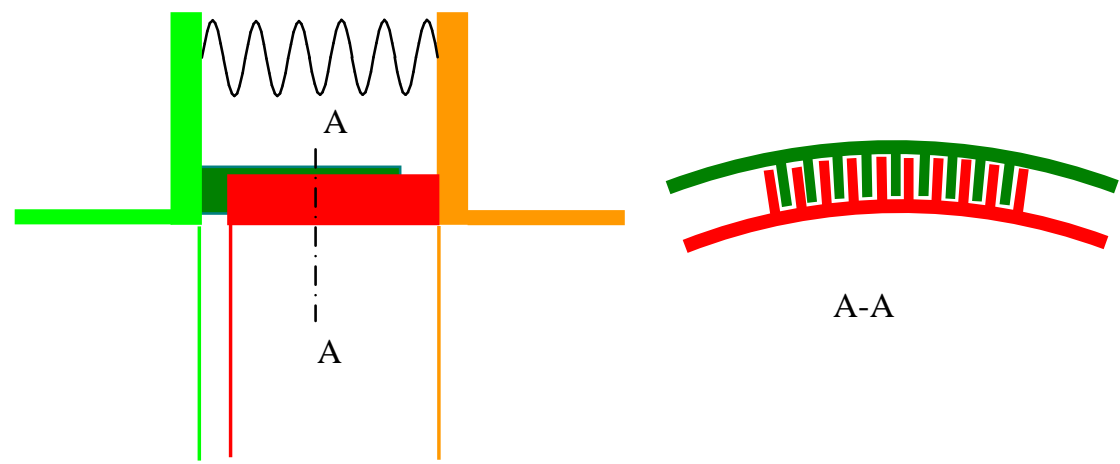

A-A

Fig. 3 Capacitor formed by two coaxial cylinders with teeth on each to enlarge capacitance -- Case 2

Now we give a numerical example. For the ERL project, the following parameters are assumed.

$\begin{array}{lll}\text { Bellows size: } & \text { Outer diameter } & 33 \mathrm{~cm} \\ & \text { Inner diameter } & 30 \mathrm{~cm} \\ & \text { Length } & 5 \mathrm{~cm} \\ \text { Beam pipe: } & \text { Inner Diameter } & 24 \mathrm{~cm} \\ \text { Charge per bunch } & 10 \mathrm{nC} \\ \text { Bunch repetition rate } & 9.383 \mathrm{Mb} / \mathrm{s} \text { (Mega bunch per second) }\end{array}$

For the above bellows structures, we choose the following geometry:

Case 1: two simple coaxial cylinders with an overlap length of $4 \mathrm{~cm}, 1 \mathrm{~mm}$ gap.

Case 2: adding teeth on the cylinders with the following parameters:

Teeth Number180, Height $9 \mathrm{~mm}$, thick $\sim 1.5 \mathrm{~mm}$, gap $0.5 \mathrm{~mm}$, overlap length $40 \mathrm{~mm}$

The expected performance data for each bellows case was calculated with the above numbers and is summarized in Table 1.

Table 1

\begin{tabular}{|l|c|c|}
\hline & Case 1 & Case 2 \\
\hline Gap of capacitor & $1 \mathrm{~mm}$ & $0.5 \mathrm{~mm}$ \\
\hline Number of teeth & 0 & 180 \\
\hline Length (Capacitance) & $4 \mathrm{~cm}$ & $4 \mathrm{~cm}$ \\
\hline \hline Capacitance & $267 \mathrm{pF}$ & $2600 \mathrm{pF}$ \\
\hline Beam induced voltage & $38 \mathrm{~V}$ & $3.8 \mathrm{~V}$ \\
\hline Beam decelerating & $19 \mathrm{~V}$ & $1.9 \mathrm{~V}$ \\
\hline Loss factor & $1.9 \times 10^{9} \mathrm{~J} / \mathrm{C}^{2}$ & $1.9 \times 10^{8} \mathrm{~J} / \mathrm{C}^{2}$ \\
& $=1.9 \times 10^{-3} \mathrm{~V} / \mathrm{pC}$ & $=1.9 \times 10^{-4} \mathrm{~V} / \mathrm{pC}$ \\
\hline Total power loss & $18 \mathrm{~W}$ & $1.8 \mathrm{~W}$ \\
\hline
\end{tabular}


The loss factors and total losses are moderate, indicating that the capacitive RF shield would be acceptable.

\section{HIGH ORDER MODES IN THE BELLOWS CHAMBER}

As far as HOMs are concerned, the above model is not detailed enough, although it exhibits the basic phenomenon. In fact, the simple L \& C circuit, shown in Fig. 1, represents only a sinusoidal oscillation, while the field pattern of the decaying oscillation inside the chamber can be much more complex. Conventionally, this pattern is regarded as a combination of all modes, including the fundamental mode and all high order modes. The latter depend strongly on the bellows geometry and the profile of the bunch.

In the case of a multi-bunch beam, if one or more modes are synchronous with the harmonics of the beam, those oscillations will be enhanced about $Q$ times ( $Q$ value is mode dependent). This is most harmful and has to be avoided.

In addition, other facts are worthy to note. (1) This mode resolution is based on linear assumption. (2) The oscillation energy must be absorbed somewhere.

In this regard, one can put a ferrite ring, or other lossy material, inside the bellows chamber. Fig. 4 shows a ferrite ring (blue) attached to the flange, which supports the bellows. Obviously, there must be flux inside the ferrite linking strongly with the discharging current. Conceivably, the oscillation will be rapidly damped by the ferrite. Since the ferrite is a nonlinear material, the HOM analysis based on the linearity assumption is no longer valid. Therefore it is necessary to verify the effect via experiment.

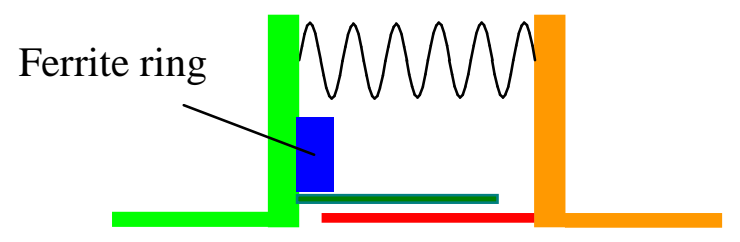

Fig. 4 A ferrite ring (blue) attached on the flange for damping

Of course, the insertion of the ferrite will change the oscillation frequency. It is interesting to note that the nonlinearity of the material in this case is favorable rather than harmful. The nonlinearity implies that the permeability depends on the field intensity and the temperature. Consequently, if the HOM frequency is synchronous with a harmonic of the beam, the intensity of the oscillation will increase gradually. Once the intensity goes up, or the temperature goes up, the permeability changes also. The latter results in a frequency shift, which implies off-resonance and thus automatically limits the final intensity. Since the discharging path is clear, to control HOM would not be difficult. 


\section{THE CONCERN OF THE BUNCH LENGTH}

We assumed that the size of the capacitor is negligible in comparison with the bunch length. Unfortunately, it is not always true. In the ERL project, the bunch length is designed to be about $9 \mathrm{~mm}(\sigma \approx 2 \mathrm{~mm})$, whereas in the above examples, the capacitor is $40 \mathrm{~mm}$ long in the beam direction, thus longer than the bunch.

In this case, when the bunch traveling along axis reaches the head of the capacitor, its image charge can not fill the whole area of the capacitor, but fill only its head part. Roughly, this equals the same length as the bunch and the capacitor acts more like a transmission line rather than a capacitance.

If the bunch travels with the speed of light, its image travels with the same speed along the capacitor. The effective capacitance is roughly:

$$
C_{\text {eff }}=\ell C_{1}
$$

where $\ell$ is the bunch length and $C_{1}$ the capacitance per unit length. Consequently, the $C$ in Eqs.(1) to (6) should be replaced by $C_{\text {eff }}$. Substituting above data, we get

$$
C / C_{\text {eff }}=40 / 9 \approx 4.4
$$

It means that the loss factor and total loss will be increased 4.4 times. The sequent oscillation may be more complex, nevertheless, the fundamental phenomenon remains.

\section{HIGH ORDER MODES IN THE CAVITY}

Previously, we discussed only the phenomenon related to the interaction between bunch and the bellows chamber. However, the RF-shield should also satisfy another requirement. In the ERL-cooling project, there is a ferrite damper located outside the cryogenic tank. Fig. 5 shows the schematic.

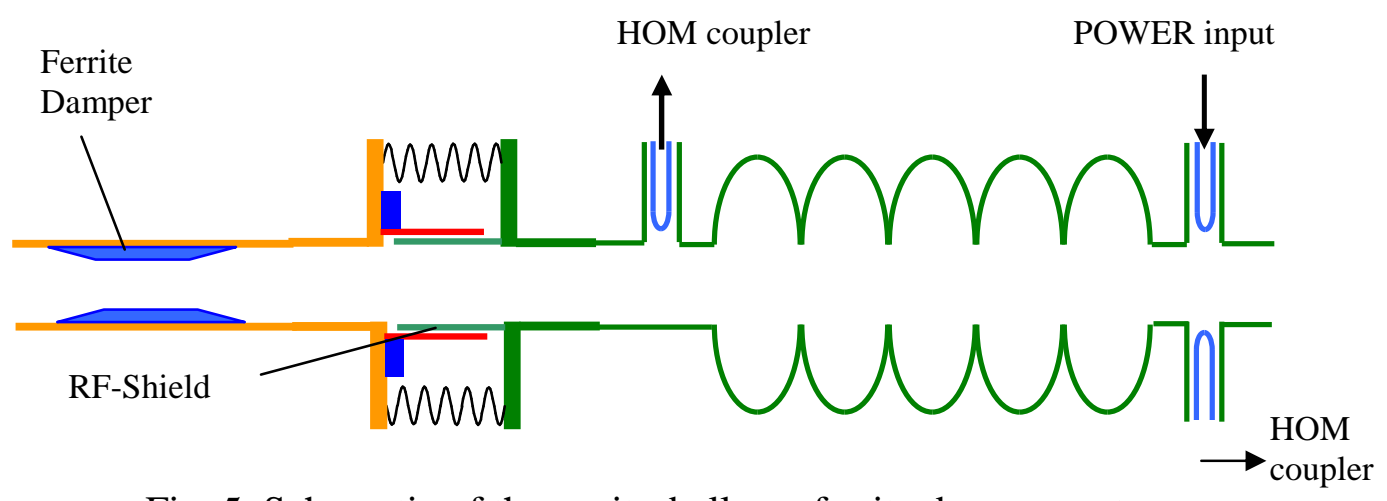

Fig. 5. Schematic of the cavity-bellows-ferrite damper system

The cavity is so designed that the beam pipe is large enough that all HOM frequencies are above the cut-off. Thus the HOMs excited inside the superconducting 
cavity travel across the RF-shield and reach the ferrite damper. The requirement is that the RF-shield does not block nor reflect the wave. Ideally it performs as a short.

Viewed from a waveguide mode, the capacitive RF-shield mentioned above is not a capacitance; rather, it is a piece of transmission line. Fig. 6 scales up the RF-shield part in Fig. 5. It consists of two coaxial cylinders. It behaves as a capacitor at low frequency, but is a coaxial line at high frequency. There is an opening in the inner surface (where the $Z_{\text {in }}$ arrow points). We have to know what the value of the input impedance, $Z_{\text {in }}$, is for each mode.

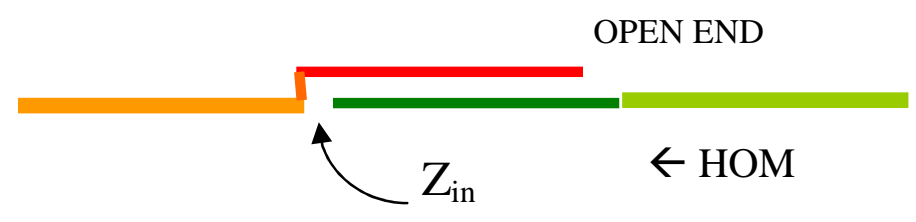

Fig.6 The effective circuit of the structure seen by HOM

Since the field pattern depends on the mode and frequency, to precisely evaluate the input impedance of the transmission line is difficult. Nevertheless, one can analyze it qualitatively and make an estimate.

The pipe is a round waveguide. The waveguide mode is either TM-mode, or TEmode, but no TEM mode exists. For TE modes, the wall current is azimuthal. So the opening has little influence. For TM modes, the wall current is longitudinal and is interrupted by the opening, so that an impedance does exist. We now consider TM modes only.

As is well known, the input impedance of a uniform transmission line is:

$$
Z_{\text {in }}=Z_{0} \cot \left(2 \pi \ell / \lambda_{g}\right)
$$

where $Z_{0}$ is the characteristic impedance, $\ell$ is the length of the line, and $\lambda_{g}$ is the waveguide wavelength. Here we assume, as one can see in Fig. 5 and Fig. 6, that the end of this line is open, or the terminal impedance is infinitive, which is, strictly speaking, not correct, but is a good approximation.

Both $Z_{0}$ and $\lambda_{g}$ are mode and frequency dependent, thus the $Z_{\text {in }}$ value is not unique. Since the wall current of the TEM mode is also longitudinal, we can use it as an approximation. For the TEM mode, the characteristic impedance can be figured out by its capacitance per unit length, i.e.

$$
Z_{0}=\ell / c C_{1}
$$

where $c$ is the speed of light. The input impedance is simply

$$
Z_{\text {in }}=Z_{0} \cot (2 \pi \ell / \lambda)
$$

The free space wavelength $\lambda$ is always less than waveguide wavelength $\lambda_{g}$, so the above equation is conservative in most cases. 
Table 2 summarizes the data of the two cases discussed above. Since the input impedance depends on the frequency, we have chosen two frequencies. According to the RF cavity simulation, the HOM with frequency above $1.6 \mathrm{GHz}$ presents little danger. Thus we chose $1.6 \mathrm{GHz}$ as upper limit.

Table 2

\begin{tabular}{|l|c|c|}
\hline & Case 1 & Case 2 \\
\hline \hline Line length & $4 \mathrm{~cm}$ & $4 \mathrm{~cm}$ \\
\hline Capacitance & $267 \mathrm{pF}$ & $2600 \mathrm{pF}$ \\
\hline Unit length Capacitance & $67 \mathrm{pF}$ & $650 \mathrm{pF}$ \\
\hline$Z_{0}$ & $0.5 \Omega$ & $0.05 \Omega$ \\
\hline $\mathrm{Z}_{\text {in }}(f=703 \mathrm{MHz})$ & $0.75 \Omega$ & $0.075 \Omega$ \\
\hline $\mathrm{Z}_{\text {in }}(f=1.6 \mathrm{GHz})$ & $2.1 \Omega$ & $0.21 \Omega$ \\
\hline
\end{tabular}

One can see that the input impedances are rather low. It implies HOMs will suffer only a small reflection.

Another question is: how the impedance behaves if there is a resonance in the bellows chamber at a particular frequency? Then the input impedance can be very large and becomes a concern. In order to minimize the risk, one can adopt two methods. (1) Per experiment, adjust the geometry such that the resonant frequency is shifted off from the HOM frequency; (2) The ferrite damper in the chamber will weaken any possible resonance.

\section{THERMAL ISOLATION ISSUE}

Thermal isolation is not a concern providing that both ends of the RF shield and bellows are at room temperature. In the case that a bellows is used in a cryostat containing a superconducting cavity to compensate the thermal shrinkage / expansion, the thermal isolation of the RF shield becomes an issue.

Intuitively, a capacitive RF shield avoids any mechanical contact. Thus it also avoids any conduction heat transfer. But, if the capacitance requires a rather large surface area between two electrodes, then the heat radiation increases. It is necessary to check if the radiation power is allowable for the cryostat.

Assuming one end is at $4 \mathrm{~K}$, then its radiation is negligible. The radiation power from the warm end to cryostat is:

$$
W_{R}=A R
$$

where $A$ is the area and $R$ the radiation coefficient given by

$$
R=\varepsilon \sigma T^{4} \quad \text { with } \quad \sigma=5.670 \times 10^{-12} \mathrm{~W} / \mathrm{cm}^{2} \mathrm{~K}^{4}
$$

Assuming $\varepsilon=0.5$, the total radiation power is summarized in Table 3

Table 3

\begin{tabular}{|c|c|c|c|}
\hline \multicolumn{2}{|c|}{} & Case 1 & Case 2 \\
\hline \hline \multicolumn{2}{|c|}{ A (Area of electrode) } & $300 \mathrm{~cm}^{2}$ & $1600 \mathrm{~cm}^{2}$ \\
\hline $\mathrm{W}_{\mathrm{R}}$ & $\mathrm{T}=300 \mathrm{~K}$ & $6.9 \mathrm{~W}$ & $37 \mathrm{~W}$ \\
\cline { 2 - 4 } (Radiation Power) & $\mathrm{T}=80 \mathrm{~K}$ & $0.035 \mathrm{~W}$ & $0.19 \mathrm{~W}$ \\
\hline
\end{tabular}


This does not include the heat transfer via the bellows. It is clear, that if the warm end is at liquid nitrogen temperature, the heat leakage is negligible, but becomes a concern if the warn end is at room temperature.

\section{References}

1. I. Ben-Zvi et al., Proc PAC 03, p.39

2. D. Wang et al., Proc PAC 03, p.1300

3. K-Y Ng, in Physics of Particle Accelerators, AIP 184 vol.1, p.473, sect. 4.4

4. Y. Suetsugu, K. Oshima, and K. Kanazawa, Rev. Sci. Instr. 67, 2796 (1996)

5. K. Kanazawa, Y. Suetsugu, "Development of a bellows assembly with RF -shield for KEKB”, Proc. EPAC96, p.1072.

6. S. Ecklund, F. J. Dacker, A. Fisher, A. Kulikov, M. Sullivan, "High order mode heating observation region", PAC01 proceedings p.3576, 2001

7. A. Novokhatski, S. Weathersby, "RF modes in the PEP-II shielded vertex bellows", PAC03 proceedings KPAG044, 2003, SLAC-PUB-9952 2003.

8. Y. Suetsugu, M. Shiral and K. Shibata, "Possibility of comb-type RF-shield structure for high current accelerators", Physical Review Special Topics, 6, 103201 (2003)

9. H. Wiedemann, Particle Accelerator Physics II, (Springer,-Verlag, Berlin, 2003), p.194 